\title{
Framework of Sensor Fusion Network for an Inverted Pendulum Mobile Robot KoRo
}

\author{
Masahiro Wada ${ }^{\dagger}$, Masahiro Tanaka ${ }^{\dagger}$, Tomohiro Umetani ${ }^{\dagger}$, \\ Takashi Kuwata $^{\dagger}$, Naomichi Kuga ${ }^{\dagger}$, Akimasa Kojima ${ }^{\dagger}$, Takayuki Matsuda ${ }^{\dagger}$, \\ Tatsuya Sakai ${ }^{\dagger}$, Naoki Adachi ${ }^{\dagger}$, Kazutoshi Inaoka ${ }^{\dagger}$ and Minoru Ito $^{\S}$ \\ $\dagger$ Dept. of Intelligence and Informatics, Konan University, \\ 8-9-1 Okamoto, Higashinada-ku, Kobe 658-8501, Japan \\ $\S$ Maizuru National College of Technology, \\ 234 Aza Shiroya, Maizuru 625-8511, Japan \\ Email: wada-m@konan-u.ac.jp
}

\begin{abstract}
In this study, a framework of fusion network with several sensors for an inverted pendulum mobile robot is investigated by developing various services as a campus robot in our university. Several devices has been equipped on the robot for sensors as detecting obstacles, walls, human body, boundary of the ground and so on. In order to implement an algorithm for safety to drive, a framework of sensor fusion network realized by RT-middleware components is explained. As one of example, we show an experimental result using the framework on the campus event.
\end{abstract}

\section{Introduction}

An inverted pendulum mobile robot is one of interesting moving platform robot. Along the increasing interest in robotics fields, the mechanism for stability of specific robots has become an important problem of researches. We know the Segway Robotics Mobility Platform (RMP) ${ }^{1}$ as an autonomous mobile robot with only two wheels which makes use of a self-balancing and a body swing mechanism based on the principle of the inverted pendulum. Two wheeled inverted pendulum robot has just two driving wheels and it has four degrees of freedom such as coordinates on twodimensional plane, direction and rotation of the robot. Several studies using two-wheeled pendulum mobile robots had been developed in the wide categories [1]-[5]. In the previous work, we first reported a unique campus robot "KoRo" (abbr. Konan Robot) using the mobile robot with several sensors based on Segway RMP200 platform [6]. Also, simultaneous localization and mapping (SLAM) is an important study or research topic for autonomous moving robots for several outdoor and indoor environments [7]-[9]. Furthermore, this model of moving robot is necessary to be considered as a stochastic model. We now are making the robot KoRo moving on our campus autonomously by using several sensors.

\footnotetext{
${ }^{1}$ http://rmp.segway.com/
}

The sensors such as a USB camera, a laser range scanner (LRS) and a thermal imaging sensor on the moving robot are good at detecting their position, and further odometer equipped on the robot is used for accuracy of them. In recent years, dynamics of autonomous moving robot in several environment will be trying to treat as probabilistic models [10]. It is very important to combine both conditions of the robot and several informations obtained from the sensors as probabilistic variables.

Therefore, it is necessary to obtain precisely their posture such as position and angles. Similarly, it should be composed their sensor informations in a sense of the sensor fusion algorithm. It is also very important to implement an algorithm for safety to drive, and then we show a framework of sensor fusion network realized by RT-middleware components in this study. We show an example of experimental result in the sense of sensor fusion network using RT-middleware on the campus event.

\section{Framework of Sensor Fusion Network using Several Devices}

The inverted pendulum mobile robot such as Segway RMP has just two wheels. It is unstable when the motor activation is turned off. Contrarily, it is usually swinging when the motor activation is turned on, hence the robot is self-balancing mode. The sensor on the robot cannot obtain the correct data when the robot is moving. Therefore, we use only several data when inclination of the robot is around zero with respect to the perpendicular line. On the other hand, this robot has two odometers for each wheel by 33215 counts per a meter. The information obtained by each odometer can be accumulated one by one. However, it is easily considered that the error margin of their information grows gradually whenever moving. Therefore, we consider that the whole data should be treated as a stochastic model. Verification for the accuracy of odometer installed in 

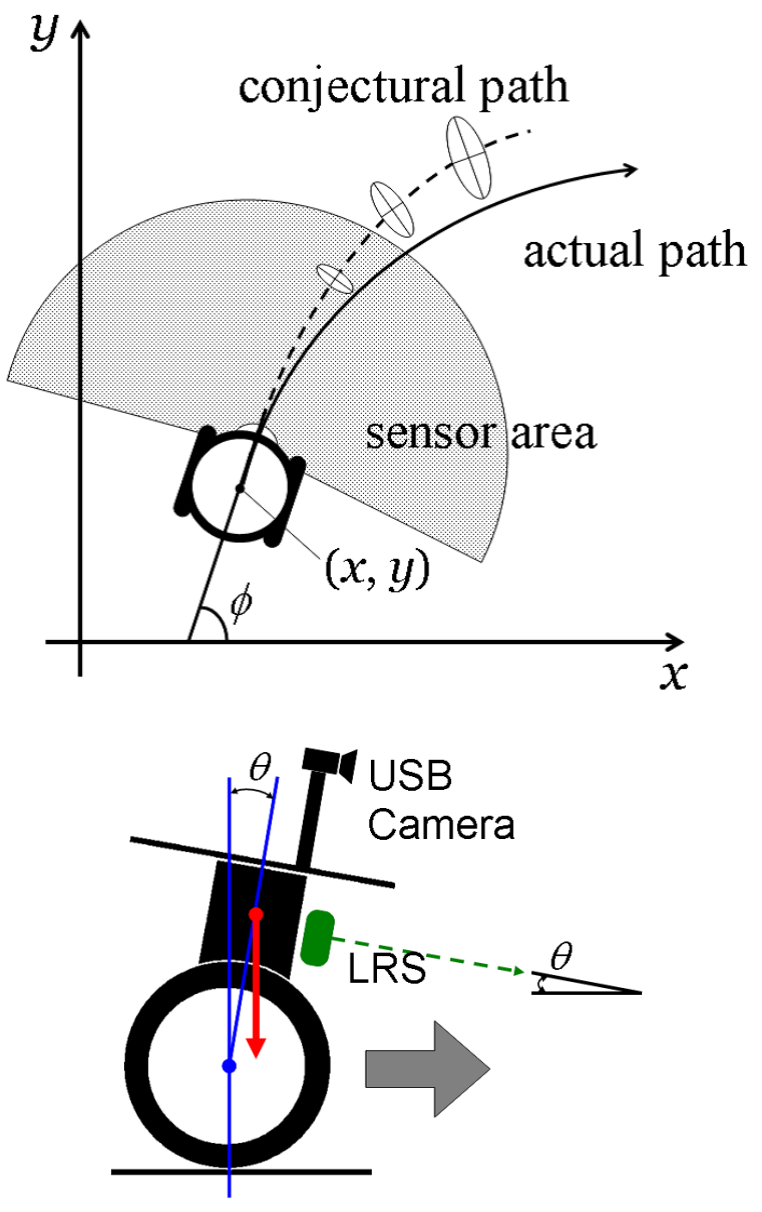

Fig. 1: Definition of coordinate and angle for the inverted pendulum mobile robot

the body is required by several measurement, then the probabilistic model should be derived and verified by the actual data. Consequently, it is possible to reduce the error margin in SLAM, and to correct sensor data.

The inverted pendulum mobile robot has an angle of unstable factors as shown in Fig. 1 with the position $(x, y)$, the angle $\phi$ and another angle $\theta$ as inclination with respect to the perpendicular line. A simple dynamical model of four degrees of freedom for two wheels inverted pendulum is derived by position, speed, heading orientation and inclination. The state space dynamical equation of the system is described in the following.

$$
\dot{\boldsymbol{x}}(t)=A \boldsymbol{x}(t)+B \boldsymbol{u}(t)+\mathbf{f}(\boldsymbol{x}, t)
$$

where $\boldsymbol{x}=[x, y, \phi, \theta], A$ and $B$ are constant matrices, and $\mathbf{f}$ is the uncertainty matrix for their nonlinearity.

At the same time, we define a probability density of their state, localization and map from observation and input for stochastic models at time $t$ in the following.

$$
p\left(x_{t}, m \mid z_{1: t}, u_{1: t}\right)
$$

In the problems concerned with SLAM, it is important to decide simultaneously both state $x_{t}$ and map $m$

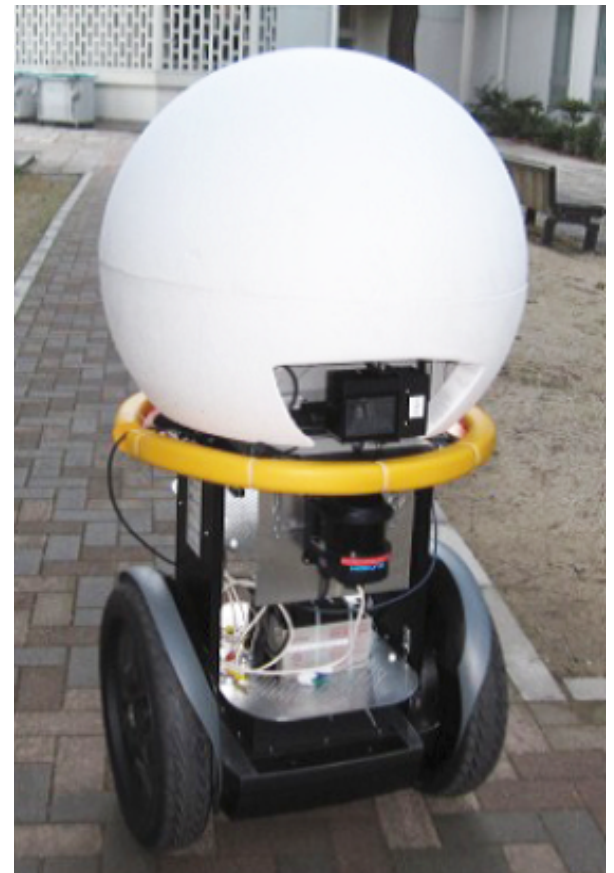

Fig. 2: General view of KoRo

which is given by observation $z$ from the sensors and input $u$, for probability density function $p$. Likelihood function of a posteriori state estimate can be calculated by several mathematical techniques such as Kalman filter (KF), EKF, Bayesian network and so on.

To simplify, a discrete model can be described for their state by several filters $G$ as follows.

$$
x_{t}=x_{t-1}+G \Delta_{u}
$$

The error margin is accumulated step by step. Therefore, it should be made amends for accuracy with several sensors.

In this study, several sensors and devices are using for their operation. Figure 2 shows a general view of the whole body KoRo with some sensors. Figures 3 and 4 show a USB camera equipped on the top and Hokuyo laser range scanner UXM-30LX-E, respectively. In order to control safely and stabilize the robot, we make an operation model using several components by RT-middleware [11]. The RT-middleware (RTM) is a software platform to construct easily several modules which can be realized to connect with various systems or devices for the robot technology. In this study, we make and compose several components both of sensors and operation block on the RTM platform for stability and safety to operate the robot as shown in Fig. 5 which is described with some operational signals and role of modules. In this figure, the robot can move automatically by a control module from several sensors such as an USB camera and an LRS device, and it can be controlled by an operator from the game controller to operate safely. At this moment, in our KoRo 


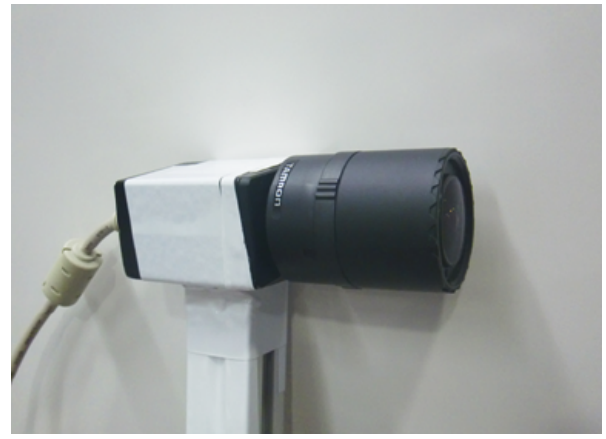

Fig. 3: USB camera equipped on the top of the body

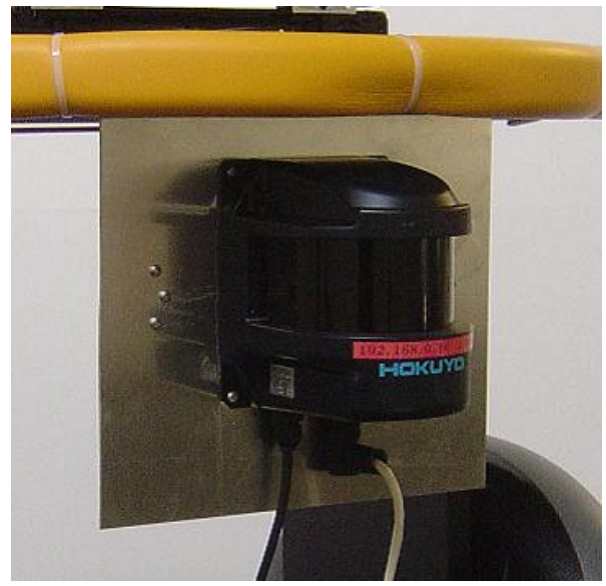

Fig. 4: Hokuyo LRS UXM-30LX-E attached at the middle point of height for the body

project, we could obtain several data from the mobile robot by using RTM. On the other hand, some error margin of movement from the mobile robot is accumulated as shown in Fig. 1 illustrated with actual and conjectural path. Our aim is to construct to reduce the error margin of the actual measurement value to the conjectural one. However, it is thought that the sensors on the mobile robot shows comparatively an excellent result.

\section{Example Model of Following Target Object with RTC modules}

We show an example of following mode to the target object on a campus event. The target object is a redcolored umbrella which is used for an easy construction instead of a human body. The robot follows the target, it is like a faithfulness dog. Figure 6 shows a diagram of four states and transition types. It has four states in the followings, then change each state by their conditions.

\section{Waiting mode}

1. Move to the direction after detecting a target (red-colored umbrella)
2. Reach to the target within $1[\mathrm{~m}]$, then transition to a following mode

Following mode

1. Move to the target if the robot can take more than any distance (e.g., $1.5[\mathrm{~m}])$, it terminates if within the distance

2. Transit to the come-back mode when the robot lost track of the target

Come-back mode

1. Go back to the appointed point

2. Turn to a basic position and transit to the waiting mode

Remote-controlled mode

1. If some troubles occur, the robot can be terminated by force signals from a wireless game controller

2 . The robot can be controlled by the game controller

We explain details of some RT components and construction of this operation model. A fundamental feature of each component is shown as follows.

[UsbCamera]

This component means the module is trying to find the target in the case of red-colored umbrella, and On the other hand, in order to transit to the comeback mode, the module then finds the other target for instance a big blue target. An algorithm to find the target is as follows:

1. Get an image

2. Convert to an HSV image

3. AND logic operation of both Hue and Saturation, then detect the target. Settings Hue is over than 300 and Saturation is over than 200.

4. Put a label separated by expanding or shrinking area, respectively

5. Remove and remain only label of the largest value

6. If it is larger than threshold value 1000, then in the case of true:

(a) Obtain marginal probability distribution for the horizontal axis

(b) Calculate a center point of the area

(c) Output detection of the target, direction and size of area. Here the image of area is divided into seven parts, select an area which is corresponding to the center point. Then, move toward any direction $25,20,10,0,-10,-20$ and -25 , respectively.

in the case of false:

(a) Output no detection of the target

7. Go to the first step

[hachi_laser]

This component is a module to output direction and obstacles from scanned data of LRS. It requires a pitch 


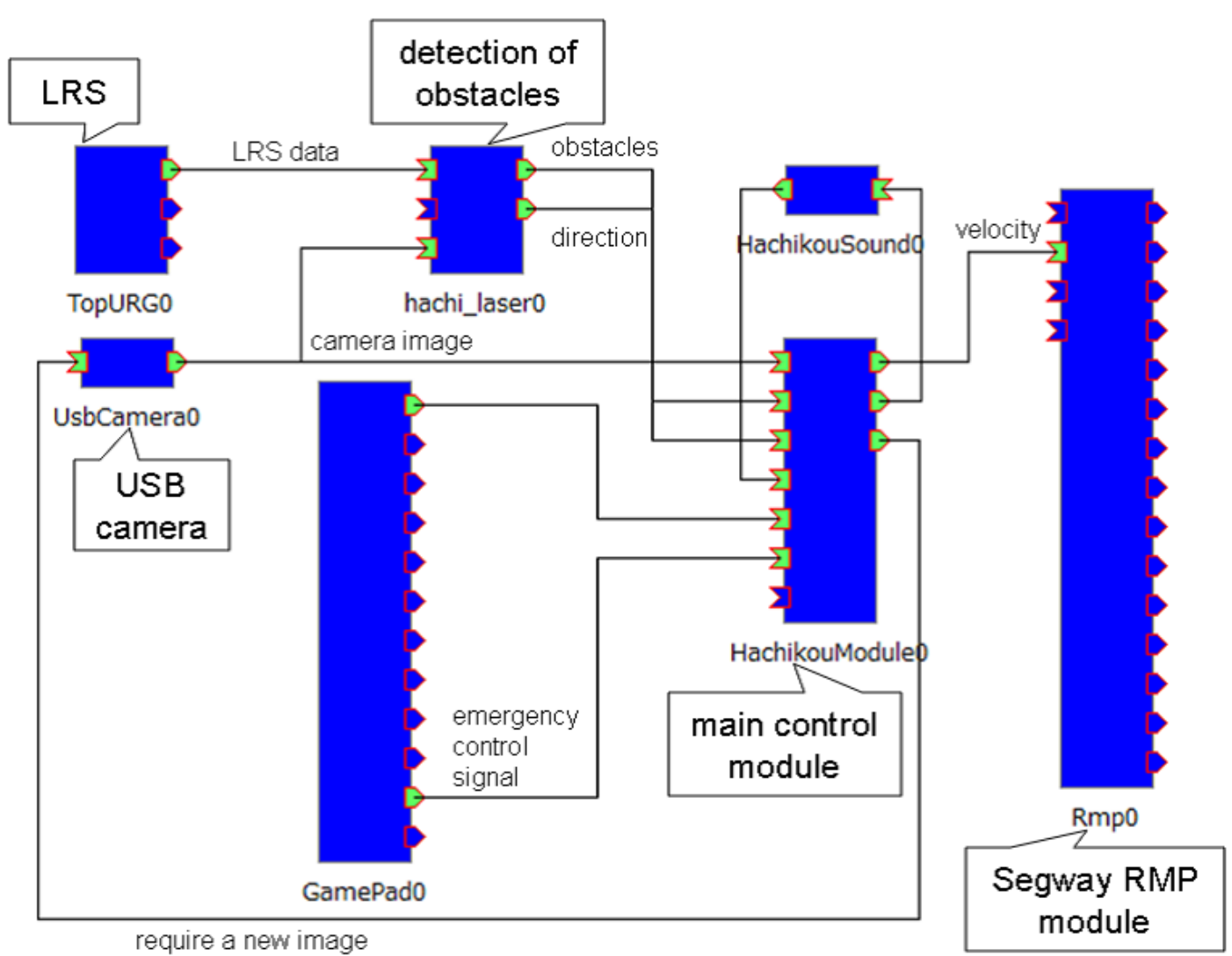

Fig. 5: Diagram of the components by RT-middleware including several sensors, main algorithms, control and sound modules

angle of the RMP and detection of the target. Because the target has already been detected, objects are regarded as obstacles for the other direction. Here, sensing area is around $180^{\circ}$ of the front side within $5[\mathrm{~m}]$ at a distance from the sensor.

\section{[HachikouModule]}

This module composes data obtained several components, then output to the RMP as velocity control. It determines operating modes as shown in Fig. 6 .

\section{[HachikouSound]}

This is an operation module which sounds a cute voice in each scene.

\section{[RMP]}

This is a module of the RMP body. It requires an input for a velocity. On the other hand, we can obtain several information such as an odometry data, a pitch angle, a roll angle and so on.

\section{[GamePad]}

This module is necessary to operate safely. It can be forcibly switched operating modes as controlled by button of the game pad. Normally, it does not require because the robot repeats automatically three states, i.e., waiting mode, following mode and come-back mode.

In this experiment for the diagram as shown in Fig. 5, the robot detects the target by camera and sensor modules, then it follows the target. Figure 7 is a snapshot when the campus event was held on our campus. KoRo detects a red-colored umbrella, then follows the target successfully. Thus, we considered that it is easy to construct the model controlled by sensor fusion network for such mobile robot on the RT-middleware environment in this way.

\section{Conclusions}

A basic framework of the autonomous two wheeled inverted pendulum mobile robot KoRo had been proposed for a sensor fusion network model. It had successfully detected some features for obstacles and human, therefore several sensors could be used in the sense of sensor fusion control. Some simulation results of the real mobile robot using the RT-middleware components had been shown in this study.

For the near future research, we will apply these techniques to the autonomous moving robot with simultaneous localization and mapping. As the other problem, it is usually unstable because this mobile robot KoRo has only two wheels for their movement, therefore it has necessary to no only control for their posture but also amend and revise sensor angles obtained from the robot by using a pan-tilt camera platform for instance. 


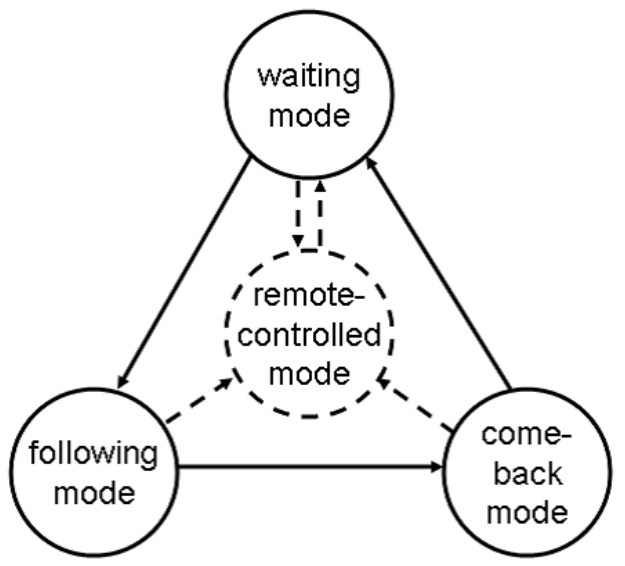

Fig. 6: Diagram of four operating modes and transition route

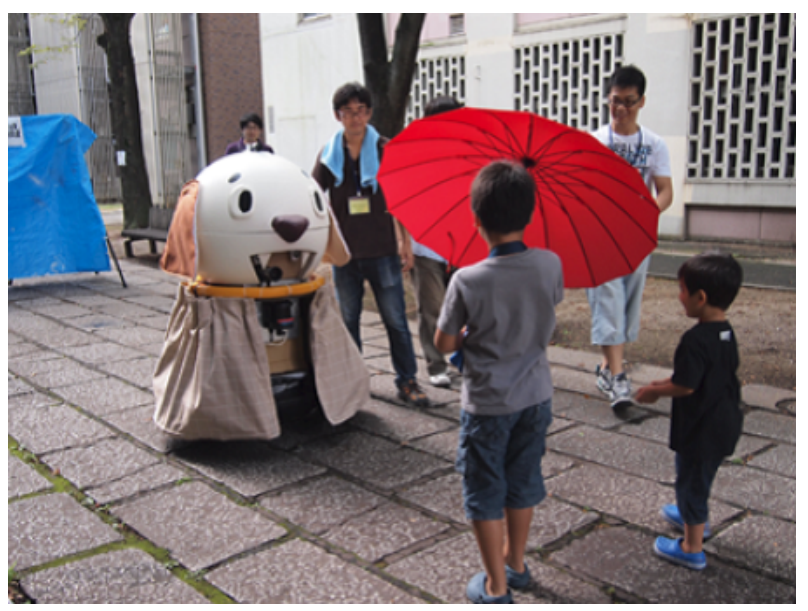

Fig. 7: Snapshot on the campus event: KoRo detected a red-colored umbrella, then it follows the target.

\section{Acknowledgment}

This research has been partially supported by "The Hirao Taro Foundation of the Konan University Association for Academic Research" and also supported in part by MEXT, Japan.

\section{References}

[1] K. Pathak and S. K. Agrawal, "Band-Limited Trajectory Planning and Tracking for Certain Dynamically Stabilized Mobile Systems," ASME Journal of Dynamic Systems, Measurement and Control, vol. 128, pp. 104-111, 2006.

[2] Y. Hosoda, S. Egawa, J. Tamamoto, K. Yamamoto, R. Nakamura and M. Togami, "Development of Human-Symbiotic Robot "EMIEW" - Design Concept and System Construction -," Journal of Robotics and Mechatronics, vol. 18, no. 2, pp. 195-202, 2006.

[3] S. W. Nawawi, M. N. Ahmad and J. H. S. Osman, "Real-Time Control of a Two-Wheeled Inverted Pendulum Mobile Robot," Proc. of World Academy os Science, Engineering and Technology, vol. 29, pp. 214-220, 2008.

[4] D. Y. Lee, Y. H. Kim, B. S. Kim, Y. K. Kwak, "Dynamics and Control of Non-holonomic Two Wheeled Inverted Pendulum Robot," the Eighth Int. Symp. on Artificial Life and Robotics (AROB8th, '03), pp. 415-418, 2003.

[5] L. Ojeda, M. Raju and J. Borenstein, "FLEXnav: A Fuzzy Logic Expert Dead-reckoning System for the Segway RMP," Proc. of the SPIE Defense and Security Symposium, Unmanned Ground Vehicle Technology, pp. 11-23, 2004.

[6] M. Wada, M. Tanaka, T. Umetani and M. Ito, "Framework of Control and Stabilization System for an Inverted Pendulum Moving Robot," Proc. of the 42nd ISCIE International Symposium on Stochastic Systems Theory and Its Applications (SSS'10), pp. 1-5, 2011.

[7] M. Tanaka, H. Hirano, S. Onishi, T. Umetani and M. Wada, "Localization of a Moving Robot by Sensor Fusion," Proc. of 2010 RISP International Workshop on Nonlinear Circuits, Communication and Signal Processing (NCSP'10), pp. 556-559, 2010.

[8] T. Kuwata, M. Tanaka, M. Wada, T. Umetani and M. Ito, "Localization of Segway RMP," Proc. of the SICE Annual Conference, pp. 1675-1680, 2011.

[9] M. Tanaka, T. Kuwata, T. Sakai, A. Kojima, N. Adachi, K. Inaoka, M. Wada, T. Umetani, M. Ito, N. Kuga and T. Matsuda, "Demonstrations of Mobile Robot - Momotaro's Dog and Hachiko Modes -," Proc. of Administration Committee of Japan Joint Automatic Control Conference, 2011(to appear).

[10] S. Thrum, W. Burgard and D. Fox, "Probabilistic Robotics," MIT Press, 2005.

[11] N. Ando, T. Suehiro, K. Kitagaki, T. Kotoku, Y. Woo-Keun, "RT-middleware: distributed component middleware for RT (robot technology)," 2005 IEEE/RSJ International Conference on Intelligent Robots and Systems (IROS 2005), pp. 3555-3560, 2005. 